\title{
CONCAVE CONFORMAL MAPPINGS AND PRE-VERTICES OF SCHWARZ-CHRISTOFFEL MAPPINGS
}

\author{
M. CHUAQUI, P. DUREN, AND B. OSGOOD \\ (Communicated by Mario Bonk)
}

\begin{abstract}
Normalized conformal mappings of the disk onto the exterior of a convex polygon are studied via a representation formula provided by Schwarz's lemma. Some conditions on the pre-vertices for corresponding SchwarzChristoffel mappings are obtained. There is a connection to finite Blaschke products that characterizes the pre-vertices and leads to a curious property of Blaschke products themselves.
\end{abstract}

\section{Concave CONFormal mappings}

A conformal, meromorphic function on the unit disk $\mathbb{D}$ is said to be a concave mapping if its image is the complement of a compact, convex set. Examples we want to consider are Schwarz-Christoffel mappings onto the exterior of convex polygons, including mappings onto the complement of finite rectilinear slits. Following standard practice, we call a set of points $c_{1}, c_{2}, \ldots, c_{n}$ on the unit circle pre-vertices if they correspond to the vertices of a polygon, convex or not, under a conformal mapping of $\mathbb{D}$ onto the interior or exterior of the polygon. Locating the pre-vertices is the problem of accessory parameters for Schwarz-Christoffel mappings, and we obtain some information on the conditions the pre-vertices satisfy.

If $f$ has the form

$$
f(z)=\frac{1}{z}+b_{0}+b_{1} z+b_{2} z^{2}+\cdots,
$$

then a necessary and sufficient condition for $f$ to be a concave mapping is

$$
1+\operatorname{Re}\left\{z \frac{f^{\prime \prime}(z)}{f^{\prime}(z)}\right\}<0, \quad|z|<1 .
$$

The simple pole at the origin guarantees that $z f^{\prime \prime}(z) / f^{\prime}(z)$ is analytic there, and

$$
z \frac{f^{\prime \prime}(z)}{f^{\prime}(z)}=-2-2 b_{1} z^{2}-6 b_{2} z^{3}+\cdots .
$$

Let us examine the condition (2) in more detail, always assuming (11). If (2) holds, then there is an analytic function $\omega$ of $\mathbb{D}$ into itself, with $\omega(0)=0$, for which

$$
1+z \frac{f^{\prime \prime}(z)}{f^{\prime}(z)}=\frac{\omega(z)+1}{\omega(z)-1} .
$$

Received by the editors April 8, 2011.

2010 Mathematics Subject Classification. Primary 30C55; Secondary 30J10.

The authors were supported in part by FONDECYT Grant \#1110321. 
Moreover, $\omega^{\prime}(0)=0$, and hence

$$
\varphi(z)=\frac{\omega(z)}{z^{2}}
$$

is analytic in $\mathbb{D}$ with $|\varphi(z)| \leq 1$ there. In terms of $\varphi$,

$$
z \frac{f^{\prime \prime}(z)}{f^{\prime}(z)}=\frac{2}{z^{2} \varphi(z)-1} \quad \text { or } \quad \frac{f^{\prime \prime}(z)}{f^{\prime}(z)}=2\left(\frac{z \varphi(z)}{z^{2} \varphi(z)-1}-\frac{1}{z}\right) .
$$

It is representation (4) that will allow us to deduce some information on pre-vertices.

Before turning to this particular problem we have several general observations. From (4) we obtain the upper and lower bounds

$$
\frac{2}{1+|z|^{2}} \leq\left|z \frac{f^{\prime \prime}(z)}{f^{\prime}(z)}\right| \leq \frac{2}{1-|z|^{2}}
$$

Equality holds in either inequality at a point $z_{0} \neq 0$ only if $\left|\varphi\left(z_{0}\right)\right|=1$, in which case $\varphi$ is a unimodular constant. Then up to a rotation and translation $f(z)=z+1 / z$, so the image of $\mathbb{D}$ is the complement of a slit of length 4 .

Next, writing

$$
|z|^{4}|\varphi(z)|^{2}\left|z \frac{f^{\prime \prime}(z)}{f^{\prime}(z)}\right|^{2}=\left|2+z \frac{f^{\prime \prime}(z)}{f^{\prime}(z)}\right|^{2}
$$

from (41) and using $|\varphi(z)|^{2} \leq 1$, we see that (2) may be replaced by the stronger inequality

$$
1+\operatorname{Re}\left\{z \frac{f^{\prime \prime}(z)}{f^{\prime}(z)}\right\} \leq-\frac{1}{4}\left(1-|z|^{4}\right)\left|z \frac{f^{\prime \prime}(z)}{f^{\prime}(z)}\right|^{2} .
$$

As above, equality holds at a nonzero point only if $\varphi$ is a unimodular constant, and so $f$ maps to the complement of a slit.

While the derivation of (5) used only $|\varphi(z)| \leq 1$, an application of the invariant form of Schwarz's lemma to (4) results in an inequality involving the Schwarzian derivative. The result is more complicated, but the case of equality is more interesting. For the Schwarzian derivative of $f$,

$$
S f=\left(\frac{f^{\prime \prime}}{f^{\prime}}\right)^{\prime}-\frac{1}{2}\left(\frac{f^{\prime \prime}}{f^{\prime}}\right)^{2}
$$

we find

$$
S f(z)=-\frac{1}{2}\left(z \frac{f^{\prime \prime}(z)}{f^{\prime}(z)}\right)^{2}\left(3 \varphi(z)+z \varphi^{\prime}(z)\right) .
$$

Near the origin

$$
S f(z)=-6 b_{1}-24 b_{2} z+\cdots .
$$

Now in (6) isolate the $\varphi^{\prime}(z)$ term and manipulate the inequality

$$
\frac{1}{2}|z|^{3}\left|\frac{f^{\prime \prime}(z)}{f^{\prime}(z)}\right|^{2}\left|\varphi^{\prime}(z)\right|\left(1-|z|^{2}\right) \leq \frac{1}{2}|z|^{3}\left|\frac{f^{\prime \prime}(z)}{f^{\prime}(z)}\right|^{2}\left(1-|\varphi(z)|^{2}\right)
$$

to lead directly to

$$
\left(1-|z|^{2}\right)\left|S f(z)+\frac{3}{2}\left(z \frac{f^{\prime \prime}(z)}{f^{\prime}(z)}\right)^{2} \varphi(z)\right|+\frac{1}{2}|z|^{3}\left|\frac{f^{\prime \prime}(z)}{f^{\prime}(z)}\right|^{2}|\varphi(z)|^{2} \leq \frac{1}{2}|z|^{3}\left|\frac{f^{\prime \prime}(z)}{f^{\prime}(z)}\right|^{2} .
$$


We get an equivalent form of (8) by multiplying through by $|z|$ and eliminating $\varphi(z)$ via

$$
z^{3} \frac{f^{\prime \prime}(z)}{f^{\prime}(z)} \varphi(z)=2+z \frac{f^{\prime \prime}(z)}{f^{\prime}(z)}=-2 b_{1} z^{2}+\cdots
$$

The result is

$$
\begin{aligned}
& \left(1-|z|^{2}\right)\left|z S f(z)+\frac{3}{2 z}\left(z \frac{f^{\prime \prime}(z)}{f^{\prime}(z)}\right)\left(2+z \frac{f^{\prime \prime}(z)}{f^{\prime}(z)}\right)\right|+\frac{1}{2|z|^{2}}\left|2+z \frac{f^{\prime \prime}(z)}{f^{\prime}(z)}\right|^{2} \\
& \leq \frac{1}{2}|z|^{2}\left|z \frac{f^{\prime \prime}(z)}{f^{\prime}(z)}\right|^{2} .
\end{aligned}
$$

From (3) and (7) we find that both sides vanish to order 2 at $z=0$, and we deduce the coefficient inequality

$$
\left|b_{1}\right|^{2}+3\left|b_{2}\right| \leq 1
$$

However, we cannot go from (10) back to (9), for instance via a Koebe transformation, because we fix the pole to be at 0 .

Each of the inequalities (2), (5), and (8) or (9) is equivalent to a mapping of the form (11) being concave. The condition (9) may be compared with a necessary and sufficient condition of Kim and Minda [3] for an analytic function to be a convex mapping of $\mathbb{D}$ :

$$
\left(1-|z|^{2}\right)^{2}|S f(z)|+2\left|\bar{z}-\frac{1}{2}\left(1-|z|^{2}\right) \frac{f^{\prime \prime}(z)}{f^{\prime}(z)}\right|^{2} \leq 2 .
$$

This was also derived in 2 as a consequence of the invariant form of Schwarz's lemma.

Equality occurs in (9) at a point $z_{0} \neq 0$ if and only if $\varphi$ is a unimodular constant, in which case $f$ is a slit mapping, or if $\varphi$ is a Möbius transformation of $\mathbb{D}$ onto itself. In the latter case, what kind of mapping is $f$ ? The answer is that $f$ is a SchwarzChristoffel mapping onto the complement of a triangle, as we will see in the next section.

\section{Pre-vertices of Concave Schwarz-Christoffel mappings}

We begin with a general geometric result on the location of the pre-vertices for a concave Schwarz-Christoffel mapping.

Theorem 1. Let $n \geq 3$. Then:

(a) Any $n$ points $c_{1}, c_{2}, \ldots, c_{n}$ on the unit circle are the pre-vertices for some conformal mapping of $\mathbb{D}$ onto the exterior of a convex $n$-gon.

(b) The points $c_{1}, c_{2}, \ldots, c_{n}$ are the pre-vertices for a conformal mapping of $\mathbb{D}$ onto the exterior of a convex n-gon, with pole at the origin, if and only if the origin is in the interior of the convex hull of $c_{1}, c_{2}, \ldots, c_{n}$.

Part (a) is a consequence of part (b) and a lemma of a topological nature. We gain a more general statement on the pre-vertices - there is no restriction on where they lie on $\partial \mathbb{D}$ - but at the cost of not knowing the location of the pole.

We give the proof of part (b) first. The necessity is a geometric interpretation of one aspect of the usual development of the Schwarz-Christoffel formula; see for 
example [4]. For a mapping $f$ of $\mathbb{D}$ onto the exterior of an $n$-gon, with pole at the origin, one has

$$
f^{\prime}(z)=C \frac{\left(z-c_{1}\right)^{2 \beta_{1}} \cdots\left(z-c_{n}\right)^{2 \beta_{n}}}{z^{2}},
$$

where $C$ is a constant, the $c_{k} \in \partial \mathbb{D}$ are the pre-vertices, and the numbers $2 \pi \beta_{k}$ are the exterior angles at the vertices of the polygon, with $\beta_{k} \neq 0$ and $-1<2 \beta_{k}<1$.

The parameters must satisfy

$$
\beta_{1}+\cdots+\beta_{n}=1, \quad \beta_{1} c_{1}+\cdots+\beta_{n} c_{n}=0 .
$$

The polygon is convex if and only if all $\beta_{k}>0$. Analytically, the second condition in (12) ensures that $f^{\prime}(z)$ has zero residue at the origin and hence that $f(z)$ is single valued. Geometrically, it says that 0 is in the convex hull of the $c_{k}$ and also in the interior of the convex hull because all the $\beta_{k}$ are strictly positive. This proves the necessity.

Conversely, suppose that $c_{1}, c_{2}, \ldots, c_{n}$ are situated on the unit circle so that

$$
\beta_{1} c_{1}+\beta_{2} c_{2}+\cdots+\beta_{n} c_{n}=0
$$

for numbers $\beta_{k}>0$ with

$$
\beta_{1}+\beta_{2}+\cdots+\beta_{n}=1 .
$$

We define the function $f^{\prime}$ by (11), and from this

$$
1+z \frac{f^{\prime \prime}(z)}{f^{\prime}(z)}=\sum_{k=1}^{n} \frac{2 \beta_{k} z}{z-c_{k}}-1 .
$$

Then (2) holds because all $\beta_{k}>0$ and $\operatorname{Re}\left\{z /\left(z-c_{k}\right)\right\}<1 / 2$. All concentric circles $|z|=r<1$ are mapped onto closed convex curves. Because the pole at the origin is simple and because $f$ is locally univalent, it follows that all such convex curves are Jordan curves. The mapping $f$ is therefore univalent in $\mathbb{D}$, and its image is the complement of a convex polygon.

This completes the poof of part (b). To deduce part (a) we need the following result.

Lemma 1. Let $c_{1}, \ldots, c_{n} \in \partial \mathbb{D}$. For any $t_{1}, \ldots, t_{n} \in(0,1 / 2)$ with $t_{1}+\cdots+t_{n}=1$ there exists a Möbius transformation $\sigma$ of $\mathbb{D}$ onto itself such that $t_{1} \sigma\left(c_{1}\right)+\cdots+$ $t_{n} \sigma\left(c_{n}\right)=0$.

With this result in hand, it is a short step to the proof of part (a) of Theorem 1. Let the points $c_{1}, c_{2}, \ldots, c_{n}$, the weights $t_{1}, t_{2}, \ldots, t_{n}$ and the Möbius transformation $\sigma$ be as in the lemma. Then by part (b) there is a conformal mapping $f$ of $\mathbb{D}$ onto the exterior of a convex $n$-gon with pole at the origin, with pre-vertices $\sigma\left(c_{1}\right), \sigma\left(c_{2}\right), \ldots, \sigma\left(c_{n}\right)$, and with exterior angles $2 \pi t_{1}, 2 \pi t_{2}, \ldots, 2 \pi t_{n}$. The mapping $f \circ \sigma$, which may no longer have a pole at the origin, meets the requirements of part (a). The proof of Theorem 1 is complete.

Proof of Lemma 1. Consider the function

$$
\Phi(z)=\sum_{k=1}^{n} t_{k} \frac{c_{k}+z}{1+\bar{z} c_{k}} .
$$

Clearly $\Phi(\mathbb{D}) \subset \mathbb{D}$, and we want to show that there exists $z_{0} \in \mathbb{D}$ for which $\Phi\left(z_{0}\right)=$ 0 . 
Let $D_{k}$ denote the closed disk

$$
\left|z-\left(t_{k}-1\right) c_{k}\right| \leq t_{k}
$$

This disk is contained in the closure of $\mathbb{D}$ and has the segment $\left[-\left(1-2 t_{k}\right) c_{k},-c_{k}\right]$ as a diameter. We observe that $\Phi(0) \notin D_{k}$ for each $k$, for

$$
\left|\Phi(0)-\left(t_{k}-1\right) c_{k}\right|=\left|c_{k}-\sum_{j \neq k} t_{j} c_{j}\right| \geq\left|c_{k}\right|-\left|\sum_{j \neq k} t_{j} c_{j}\right|>1-\sum_{j \neq k} t_{j}=t_{k} .
$$

Now consider the images $\Phi\left(C_{r}\right)$ of concentric circles $|z|=r<1$. It is not difficult to show that the curves $\Phi\left(C_{r}\right)$ converge uniformly to $\partial \mathbb{D} \cup \partial D_{1} \cup \cdots \cup \partial D_{n}$ as $r \rightarrow 1$. In fact, as $r \rightarrow 1, \Phi\left(r e^{i \theta}\right) \rightarrow e^{i \theta}$ uniformly in $e^{i \theta}$ on compact subsets of $\partial \mathbb{D}$ away from $-c_{1}, \ldots,-c_{n}$. For $r$ close to 1 and fixed $\epsilon>0$, small $\operatorname{arcs}\left\{-r e^{i t} c_{k},|t|<\epsilon\right\}$ centered near $-c_{k}$, are mapped by $\Phi$ onto arcs that are very close to tracing the entire boundary $\partial D_{k}$. Since $0 \notin D_{k}$ for each $k$, it follows by continuity that 0 must lie in the range of $\Phi$.

This lemma is a discrete version of the balanced metric construction in [5]. There it is shown, also by a topological argument, that if $\lambda \in L^{2}(\partial \mathbb{D})$, then there is a Möbius transformation $\sigma$ of $\mathbb{D}$ onto itself such that

$$
\int_{0}^{2 \pi} \lambda\left(\sigma\left(e^{i \theta}\right)\right)\left|\sigma^{\prime}\left(e^{i \theta}\right)\right| e^{i \theta} d \theta=0 .
$$

We can obtain more information about the pre-vertices through a connection to Blaschke products.

Theorem 2. Points $c_{1}, c_{2}, \ldots, c_{n}$ on the unit circle are the pre-vertices for a mapping $f$ onto the exterior of a convex n-gon, with pole at the origin, if and only if there is a finite Blaschke product $B$ of degree $n-2$ for which the $c_{k}$ are the solutions of $z^{2} B(z)=1$.

Note that this then provides a third equivalent condition in part (b) of Theorem 1

Proof. We prove sufficiency first. Let $a_{1}, a_{2}, \ldots, a_{n-2} \in \mathbb{D}$ and let

$$
B(z)=\zeta \prod_{k=1}^{n-2} \frac{z+a_{k}}{1+\overline{a_{k}} z}, \quad|\zeta|=1 .
$$

The function $z^{2} B(z)$ has degree $n$, and the $n$ solutions to $z^{2} B(z)=1$ can only lie on the unit circle. Since $\arg \left\{z^{2} B(z)\right\}$ is monotonic on $\partial \mathbb{D}$ the solutions are distinct, say $c_{1}, c_{2}, \ldots, c_{n}$. Now define the mapping $f$ of $\mathbb{D}$, with a simple pole at 0 , through the representation (4), i.e., by

$$
\frac{f^{\prime \prime}(z)}{f^{\prime}(z)}=\frac{2}{z\left(z^{2} B(z)-1\right)}=2\left(\frac{z B(z)}{z^{2} B(z)-1}-\frac{1}{z}\right) .
$$

We want to show that $f$ maps $\mathbb{D}$ onto the complement of a convex $n$-gon with $c_{1}, c_{2}, \ldots, c_{n}$ as the pre-vertices.

The first term on the right-hand side of (13) is a rational function with simple poles at the $c_{k}$, and we can write

$$
\frac{z B(z)}{z^{2} B(z)-1}=\sum_{k=1}^{n} \frac{\beta_{k}}{z-c_{k}}
$$


for some complex constants $\beta_{k}$. Then

$$
\frac{z \prod_{k=1}^{n-2}\left(z+a_{k}\right)}{z^{2} \prod_{k=1}^{n-2}\left(z+a_{k}\right)-\bar{\zeta} \prod_{k=1}^{n-2}\left(1+\overline{a_{k}} z\right)}=\sum_{k=1}^{n} \frac{\beta_{k}}{z-c_{k}} .
$$

The left-hand side is the ratio of two monic polynomials, the numerator of degree $n-1$ and the denominator of degree $n$. We need several identities. Comparing constant terms in the denominators of the two sides we have

$$
\prod_{k=1}^{n} c_{k}=(-1)^{n+1} \bar{\zeta}
$$

and comparing leading terms in the numerators we have

$$
\sum_{k=1}^{n} \beta_{k}=1 \text {. }
$$

There is no constant term in the numerator on the left. Hence, from the expression on the right,

$$
\sum_{k=1}^{n}\left(\beta_{k} \prod_{j \neq k} c_{j}\right)=0
$$

But from (15),

for any $k$; hence

$$
\prod_{j \neq k} c_{j}=\frac{1}{c_{k}}(-1)^{n+1} \bar{\zeta}=\overline{c_{k}}(-1)^{n+1} \bar{\zeta}
$$

$$
\sum_{k=1}^{n} \beta_{k} \overline{c_{k}}=0
$$

We claim that all $\beta_{k}$ are real. To show this, let $\beta_{k}=u_{k}+i v_{k}$ and consider

$$
\frac{z^{2} B(z)}{z^{2} B(z)-1}=\sum_{k=1}^{n} \frac{\beta_{k} z}{z-c_{k}}=\sum_{k=1}^{n} \frac{u_{k} z}{z-c_{k}}+i \sum_{k=1}^{n} \frac{v_{k} z}{z-c_{k}} .
$$

Equation (16) gives $u_{1}+u_{2}+\cdots u_{n}=1$, and if $z \in \partial \mathbb{D} \backslash\left\{c_{1}, \ldots, c_{n}\right\}$, then

$$
\begin{aligned}
\frac{1}{2} & =\operatorname{Re}\left\{\frac{z^{2} B(z)}{z^{2} B(z)-1}\right\}=\sum_{k=1}^{n} u_{k} \operatorname{Re}\left\{\frac{z}{z-c_{k}}\right\}-\sum_{k=1}^{n} v_{k} \operatorname{Im}\left\{\frac{z}{z-c_{k}}\right\} \\
& =\frac{1}{2} \sum_{k=1}^{n} u_{k}-\sum_{k=1}^{n} v_{k} \operatorname{Im}\left\{\frac{z}{z-c_{k}}\right\} \\
& =\frac{1}{2}-\sum_{k=1}^{n} v_{k} \operatorname{Im}\left\{\frac{z}{z-c_{k}}\right\} .
\end{aligned}
$$

We conclude that

$$
\sum_{k=1}^{n} v_{k} \operatorname{Im}\left\{\frac{z}{z-c_{k}}\right\}=0
$$

for all such $z$ on the unit circle. But

$$
\left|\operatorname{Im}\left\{\frac{z}{z-c_{k}}\right\}\right| \rightarrow \infty
$$

when $z \rightarrow c_{k}$; hence each $v_{k}$ must vanish. This proves that all $\beta_{k}$ 's are real. 
From equations (13) and (14) we obtain

$$
f^{\prime}(z)=C \frac{\left(z-c_{1}\right)^{2 \beta_{1}} \cdots\left(z-c_{n}\right)^{2 \beta_{n}}}{z^{2}},
$$

which, in light of (16) and (17) and knowing that the $\beta_{k}$ are real, corresponds to a mapping onto the exterior of a polygon. Finally, from (13) the function $f$ satisfies (2), so the mapping is injective and concave. Thus the polygon is convex and all $\beta_{k}>0$.

For necessity, suppose that $f$ is a mapping of $\mathbb{D}$ onto the exterior of a convex $n$-gon, with pole at the origin. Then (4) holds for some holomorphic function $\varphi: \mathbb{D} \rightarrow \mathbb{D}$. Moreover, $f$ also has the form as in (11), and so

$$
\frac{z^{2} \varphi(z)}{z^{2} \varphi(z)-1}=\sum_{k=1}^{n} \frac{\beta_{k} z}{z-c_{k}}=r(z),
$$

with $\beta_{k}>0$. Then

$$
z^{2} \varphi(z)=\frac{r(z)}{r(z)-1}
$$

hence $\varphi$ is a rational function. Now,

$$
\operatorname{Re}\{r(z)\} \rightarrow \frac{1}{2}
$$

when $z$ approaches $\partial \mathbb{D} \backslash\left\{c_{1}, c_{2}, \ldots, c_{n}\right\}$, while $r(z) \rightarrow \infty$ when $z$ approaches any of the $c_{k}$. Thus $r(z)$ tends to the circle $\{w: \operatorname{Re} w=1 / 2\} \cup\{\infty\}$ on the Riemann sphere as $|z| \rightarrow 1$. Therefore the function $w=z^{2} \varphi(z)=r(z) /(r(z)-1)$ tends to $|w|=1$ as $|z| \rightarrow 1$. From the reflection principle it follows that the rational function $\varphi(z)$ is a finite Blaschke product, and from the partial fraction representation we see that it has degree $n-2$. The pre-vertices are the roots of the equation $z^{2} \varphi(z)=1$.

From the proof of Theorem 2 we can extract the following corollary.

Corollary 1. If $f$ is a concave conformal mapping of $\mathbb{D}$ with pole at the origin, then, with reference to representation (4), the function $\varphi$ is a finite Blaschke product of degree $n-2$ if and only if $f$ is a mapping onto the exterior of a convex n-gon.

Specializing further we then also have

Corollary 2. A concave conformal mapping with pole at the origin, and $\varphi$ as in (4), maps $\mathbb{D}$ onto the complement of a triangle if and only if $\varphi$ is a Möbius transformation of $\mathbb{D}$ onto itself.

Finally, rounding out the discussion of the inequality (9), we have

Corollary 3. Equality holds in (9) at a single point if and only if $f$ maps $\mathbb{D}$ onto the complement of a finite slit or a triangle. In either case, equality in (9) holds everywhere.

Proof. Equality in (9) at a single point holds if and only if we have equality in the Schwarz lemma at that point. Thus either $\varphi$ is a constant of absolute value 1 or it is a Möbius transformation of $\mathbb{D}$ onto itself. In the former case, $f$ maps onto the complement of a slit, and in the latter, onto the complement of a triangle. The reverse implication also follows from Corollary 2. The final statement is a further consequence of the case of equality in Schwarz's lemma. 
One can easily show for $c_{1}, c_{2}, c_{3}$ on the unit circle that 0 is in the interior of the convex hull of the $c$ 's if and only if

$$
\left|c_{1}+c_{2}+c_{3}\right|<1 \text {. }
$$

We can be a little more explicit. The mapping $f$ onto the complement of a triangle has, as in (4),

$$
\varphi(z)=\zeta \frac{z+a}{1+\bar{a} z}
$$

for some $|\zeta|=1$ and $|a|<1$. The pre-vertices $c_{1}, c_{2}, c_{3}$ are solutions of $z^{2} \varphi(z)=1$, which are the roots of the cubic

$$
\zeta z^{3}+a \zeta z^{2}-\bar{a} z-1=0 .
$$

If we normalize the pre-vertices to satisfy $c_{1} c_{2} c_{3}=1$, then $\zeta=1$ and

$$
a=-\left(c_{1}+c_{2}+c_{3}\right) \text {. }
$$

In this case the pre-vertices determine the angles $\beta_{1}, \beta_{2}, \beta_{3}$. For the angles $\beta_{k}$ are the weights in the convex combination

$$
\beta_{1} c_{1}+\beta_{2} c_{2}+\beta_{3} c_{3}=0, \quad \beta_{1}+\beta_{2}+\beta_{3}=1,
$$

and, for a triangle, there is only one way to express a given interior point as a convex combination of the vertices.

In 2] we proved an analog of Theorem 2 for mappings onto the interior of convex polygons, though we did not explicitly note the connection with pre-vertices. We state the result as follows: there is no normalization at the origin, where the function is analytic, and the polygon can be bounded or unbounded.

Theorem 3. Points $c_{1}, c_{2}, \ldots, c_{n}$ on the unit circle are the pre-vertices for a conformal mapping $f$ onto the interior of a convex $n$-gon if and only if there is a finite Blaschke product of degree $n-1$ for which the $c_{k}$ are solutions of $z B(z)=1$.

The proof is based on the fact, established in [2, that such mappings are characterized by the representation

$$
\frac{f^{\prime \prime}(z)}{f^{\prime}(z)}=\frac{2 B(z)}{1-z B(z)}
$$

where $B(z)$ is a finite Blaschke product. The Schwarz-Christoffel formula has the form

$$
\frac{f^{\prime \prime}(z)}{f^{\prime}(z)}=-2 \sum_{k=1}^{n} \frac{\beta_{k}}{z-c_{k}}
$$

for $\beta_{k}>0$ with

$$
\sum_{k=1}^{n} \beta_{k}=1 .
$$

To our knowledge, credit for using Blaschke products to study concave conformal mappings goes to Bhowmik, Ponnusamy and Wirths. See for example [1] and the references there for other interesting work of the authors. Their starting point, goals, and results are quite different from what we do here. The authors consider univalent, concave mappings with pole at a point $p \in(0,1)$, normalized by $f(0)=0, f^{\prime}(0)=1$, and they are interested in coefficient inequalities and other results analogous to those for univalent functions. One might try formally to deduce Theorem 2 as a limiting case of the results in [1] with $p \rightarrow 0$. However, the 
normalization cannot be maintained and an extra argument is necessary, if one is possible. Moreover, the method in the proof of Theorem 2 (and Theorem 3) can also be applied to obtain the following partial results on the pre-vertices of arbitrary Schwarz-Christoffel mappings.

Theorem 4. Let $f$ be a Schwarz-Christoffel mapping of $\mathbb{D}$ with pre-vertices $c_{1}, c_{2}$, $\ldots, c_{n}$ on the unit circle. Then:

(a) If $f$ maps $\mathbb{D}$ onto the interior of an $n$-gon, then there are finite Blaschke products $B_{1}, B_{2}$ of degrees $d_{1}, d_{2}$, respectively, with $d_{1}+d_{2}=n-1$ for which the $c_{k}$ are solutions of $z B_{1}(z)=B_{2}(z)$.

(b) If $f$ maps $\mathbb{D}$ onto the exterior of an $n$-gon, with pole at the origin, then there are finite Blaschke products $B_{1}, B_{2}$ of degrees $d_{1}, d_{2}$, respectively, with $d_{1}+d_{2}=$ $n-2$ for which the $c_{k}$ are solutions of $z^{2} B_{1}(z)=B_{2}(z)$.

In either case, the polygon is convex if and only if $d_{2}=0$.

Proof. We prove part (b); the proof of part (a) runs along the same lines. To recall, the mapping $f$ has the form in (11),

$$
f^{\prime}(z)=C \frac{\left(z-c_{1}\right)^{2 \beta_{1}} \cdots\left(z-c_{n}\right)^{2 \beta_{n}}}{z^{2}}, \quad z \frac{f^{\prime \prime}(z)}{f^{\prime}(z)}=\sum_{k=1}^{n} \frac{2 \beta_{k} z}{z-c_{k}}-2,
$$

with $-1<2 \beta_{k}<1$. Let $\varphi(z)$ be the meromorphic function

$$
\varphi(z)=\frac{2+z \frac{f^{\prime \prime}(z)}{f^{\prime}(z)}}{z^{3} \frac{f^{\prime \prime}(z)}{f^{\prime}(z)}}
$$

which is derived from setting

$$
z \frac{f^{\prime \prime}(z)}{f^{\prime}(z)}=\frac{2}{z^{2} \varphi(z)-1}
$$

as suggested by (13). Then

$$
\frac{z^{2} \varphi(z)}{z^{2} \varphi(z)-1}=\sum_{k=1}^{m} \frac{\beta_{k} z}{z-c_{k}}=r(z)
$$

is a rational function with poles at the $c_{k}$. As before, on the unit circle but away from the poles, we have $\operatorname{Re}\{r(z)\}=1 / 2$. Then since

$$
z^{2} \varphi(z)=\frac{r(z)}{r(z)-1}
$$

we have for all $|z|=1$ that $\left|z^{2} \varphi(z)\right|=1$. Hence $\varphi$ is of the form $\varphi=B_{1} / B_{2}$ for finite Blaschke products $B_{1}$ and $B_{2}$. The equation $z^{2} \varphi(z)=1$ has $n$ roots, and hence the degrees $d_{1}$ and $d_{2}$ of $B_{1}$ and $B_{2}$ must satisfy $d_{1}+d_{2}=n-2$.

Finally, if $d_{2}=0$ we know that $f$ maps $\mathbb{D}$ onto the exterior of a convex polygon. Conversely, if $f$ maps $\mathbb{D}$ onto the exterior of a convex polygon, then $\varphi$ is holomorphic in $\mathbb{D}$, and hence $d_{2}=0$.

The proof of part (a) is similar, mimicking the representation (18).

Remark. We cannot claim the converse statements in parts (a) and (b) in Theorem 4 because, to take part (a), we have no way of showing that a mapping defined through (20) is injective, where $\varphi$ is a ratio of Blaschke products. We have also been unable 
to relate the degree of $B_{2}$, in either case, to the number of vertices in the polygon where the interior angle exceeds $\pi$. In particular, we have been unable to determine whether the degree $d_{2}$ and the number of obtuse angles in the polygon coincide.

\section{BlaschKe CuRVes}

The connection between Blaschke products and Schwarz-Christoffel mappings as expressed through (13) or (18) allows us to deduce a curious property of Blaschke products themselves.

Theorem 5. Let $B$ be a finite Blaschke product of degree $n \geq 1$ and let $m \geq 0$ be an integer. Let $R(\gamma)$ be the ray $r e^{i \gamma}, r>0$. Then:

(a) The curve $\theta \mapsto e^{(m+1) i \theta} B^{\prime}\left(e^{i \theta}\right)$ intersects $R(\gamma)$ exactly when $e^{i m \theta} B\left(e^{i \theta}\right)=$ $e^{i \gamma}$, and this occurs for exactly $n+m$ points on $\partial \mathbb{D}$.

(b) If $x_{1}(\gamma), x_{2}(\gamma), \ldots, x_{n+m}(\gamma)$ are the points of intersection, then

$$
\sum_{k=1}^{n+m} \frac{1}{m+x_{k}(\gamma)}= \begin{cases}1, & m \geq 1 \\ \frac{1-|B(0)|^{2}}{\left|1-e^{-i \gamma} B(0)\right|^{2}}, & m=0 .\end{cases}
$$

Proof. To ease notation we give the proof for $\gamma=0$, i.e., when $R$ is the positive real axis. The argument for the general case is the same.

The function $z^{m+1} B^{\prime}(z)$ is holomorphic in $\mathbb{D}$ and has $m+n$ zeros there. The curve $\theta \mapsto e^{(m+1) i \theta} B^{\prime}\left(e^{i \theta}\right)$ must therefore intersect the positive real axis at least $m+n$ times. Since

the identity

$$
z \frac{B^{\prime}(z)}{B(z)}>0, \quad|z|=1
$$

$$
e^{(m+1) i \theta} B^{\prime}\left(e^{i \theta}\right)=e^{i m \theta} B\left(e^{i \theta}\right)\left(e^{i \theta} \frac{B^{\prime}\left(e^{i \theta}\right)}{B\left(e^{i \theta}\right)}\right)
$$

shows that whenever $e^{(m+1) i \theta} B^{\prime}\left(e^{i \theta}\right)>0$ the quantity $e^{i m \theta} B\left(e^{i \theta}\right)$ is also positive and is thus equal to 1 . This occurs at exactly $m+n$ points on $\partial \mathbb{D}$, say $c_{1}, c_{2}, \ldots, c_{m+n}$. This proves part (a).

For part (b), first take $m \geq 1$. We use the representation formula (18) for convex Schwarz-Christoffel mappings with Blaschke product $z^{m-1} B(z)$ to write

$$
\frac{f^{\prime \prime}(z)}{f^{\prime}(z)}=\frac{2 z^{m-1} B(z)}{1-z^{m} B(z)}=-2 \sum_{k=1}^{m+n} \frac{\beta_{k}}{z-c_{k}} .
$$

Calculating the residue at $c_{k}$ we see that

$$
\beta_{k}=\frac{c_{k}^{m-1} B\left(c_{k}\right)}{\left(z^{m} B\right)^{\prime}\left(c_{k}\right)}=\frac{1}{m+x_{k}},
$$

where we have used $c_{k}^{m} B\left(c_{k}\right)=1$ and $c_{k}^{m+1} B^{\prime}\left(c_{k}\right)=x_{k}$. Then

$$
1=\sum_{k=1}^{m+n} \beta_{k}=\sum_{k=1}^{m+n} \frac{1}{m+x_{k}} .
$$

Now take the case $m=0$. Consider the partial fractions decomposition

$$
\frac{B(z)}{z(B(z)-1)}=\sum_{k=1}^{n} \frac{\beta_{k}}{z-c_{k}}+\frac{\alpha}{z} \text {. }
$$


For the residue at $c_{k}$ we have

$$
\beta_{k}=\frac{B\left(c_{k}\right)}{c_{k} B^{\prime}\left(c_{k}\right)}=\frac{1}{x_{k}}
$$

while

Next, with

$$
\alpha=\frac{B(0)}{B(0)-1}
$$

$$
B(z)=\prod_{k=1}^{n} \frac{z+a_{k}}{1+\overline{a_{k}} z}=\frac{p(z)}{q(z)}
$$

it follows from the partial fractions expression (22) that

$$
z(p(z)-q(z))=(1-\overline{B(0)}) z \prod_{k=1}^{n}\left(z-c_{k}\right) .
$$

Using this, we equate the coefficients of $z^{n}$ in the numerator of (22) to obtain

$$
\frac{1}{1-\overline{B(0)}}=\sum_{k=1}^{n} \beta_{k}+\alpha=\sum_{k=1}^{n} \beta_{k}+\frac{B(0)}{B(0)-1} .
$$

This gives

$$
\sum_{k=1}^{n} \beta_{k}=\sum_{k=1}^{n} \frac{1}{x_{k}}=\frac{1}{1-\overline{B(0)}}+\frac{B(0)}{1-B(0)}=\frac{1-|B(0)|^{2}}{|1-B(0)|^{2}} .
$$

This completes the proof of part (b).

Note that in the case $m=0$ the sum can now become arbitrarily large if $B(0)$ is real and close to $e^{i \gamma}$, and it can become arbitrarily small if $B(0)$ is close to a point on $\partial \mathbb{D}$ away from $e^{i \gamma}$. In the latter case all the $x_{k}(\gamma)$ must tend to $\infty$.

\section{REFERENCES}

1. B. Bhowmik, S. Ponnusamy, and K.-J. Wirths, Concave functions, Blaschke products, and polygonal mappings, Siberian Math. J. 50 (2009), 609-615. MR2583615 (2011a:30123)

2. M. Chuaqui, P. Duren, and B. Osgood, Schwarzian derivatives of convex mappings, Ann. Acad. Sci. Fenn. Math. 36 (2011), 449-460.

3. S.-A. Kim and D. Minda, The hyperbolic and quasihyperbolic metrics in convex regions, J. Analysis 1 (1993), 109-118. MR1230512(94h:30005)

4. A.I. Markushevich, Theory of Functions of a Complex Variable, Vol. 3, Prentice Hall, Englewood Cliffs, NJ, 1967. MR0215964(35:6799)

5. B. Osgood, R. Phillips, and P. Sarnak, Compact sets of isospectral surfaces, J. Funct. Analysis 80 (1988), 212-234. MR960229 (90d:58160)

Facultad de Matemáticas, P. Universidad Católica de Chile, Casilla 306, Santiago 22, Chile

E-mail address: mchuaqui@mat.puc.cl

Department of Mathematics, University of Michigan, Ann Arbor, Michigan 481091043

E-mail address: duren@umich.edu

Department of Electrical Engineering, Stanford University, Stanford, California 94305-9510

E-mail address: osgood@stanford.edu 